\title{
Beneficial effects of voluntary over forced exercise on skeletal muscle structure and myokines' expression
}

\author{
M.A. Eldomiaty ${ }^{1,2}$, A. Elayat ${ }^{3}$, S. Alii, ${ }^{4}$, S. Algaidi ${ }^{6}$, M. Elnaggar ${ }^{7}$ \\ ${ }^{1}$ Department of Anatomy, Faculty of Medicine, Taibah University, Saudi Arabia \\ 2Department of Anatomy, Faculty of Medicine, Tanta University, Egypt \\ ${ }^{3}$ Department of Anatomy, Faculty of Dentistry, Modern Sciences and Arts University, Egypt \\ ${ }^{4}$ Department of Anatomy, Faculty of Medicine, King Abdulaziz University, Saudi Arabia \\ ${ }^{5}$ Yousef Abdullatif Jameel Chair of Prophetic Medical Applications, Saudi Arabia \\ ${ }^{6}$ Department of Anatomy, College of Medicine, Taibah University, Almadinah Almunawwarah, Saudi Arabia \\ 'Orthopedic Surgery, Ministry of Interior Hospitals, Cairo, Egypt
}

[Received: 21 October 2019; Accepted: 21 November 2019]

Background: Myokines, a group of small proteins - mainly cytokines, are released by myocytes during muscular contraction and proved to have many biological effects locally or at systemic levels. The main objective was to study the morphological alterations and myokines expression in rat gastrocnemius muscle following forced compared to voluntary muscle contraction.

Materials and methods: Thirty-six adult male Wistar rats were divided into three groups: control, voluntary exercise and forced swimming regimen. The experiment last for 3 weeks. The weight of rats and serum corticosterone levels were recorded. The gastrocnemius muscle samples were processed for histological and immunohistochemical study of different myokines.

Results: The mean weight of rats showed no statistical difference between groups. Corticosterone level significantly increased after forced exercise. Voluntary exercise muscle fibres appeared hypertrophied with prominent transverse banding and dominating satellite cells. Forced exercise muscle showed atrophied widely spaced muscle fibres and inflammatory cell infiltrate. Voluntary exercise significantly increased optic density of interleukin 6, macrophage inhibitory and brain derived neurotrophic factors, whereas the forced exercise group showed significant decrease in their optic densities. The optic density of vascular endothelial growth factor significantly decreased in the forced exercise group. Forced exercise could be harmful to the skeletal muscle fibres and it decreases the secretion of important myokines. Further, forced exercise significantly increases the serum corticosterone level.

Conclusions: The use of exercise for the attainment of healthy life style or in psycho- or neuro-therapy should follow a thoroughly studied programme for welfare of human health. (Folia Morphol 2020; 79, 2: 350-358)

Key words: forced exercise, voluntary exercise, skeletal muscle, myokines, corticosterone 


\section{INTRODUCTION}

The emerging knowledge that skeletal muscles have a secretory function has opened an immense and exciting field in the study of muscles. The contracting muscle motivates production and secretion of many cytokines that are called myokines. Though some of these myokines were described as pro-inflammatory cytokines $[22,23]$, myokines are lately branded as potential applicants for treating metabolic and chronic diseases associated with a sedentary life-style [22].

Interleukin 6 (IL-6) is the first cytokine to be identified in the blood stream during muscle contractions. It is produced and released from the skeletal muscles in significant levels after prolonged exercise and produced pleiotropic functions in different tissues of the body [17]. However, contradictory harmful effects of IL- 6 as promotion of atrophy and muscle wasting have been proposed [16].

Vascular endothelial growth factor (VEGF) is considered as the utmost important pro-angiogenic cytokine in most tissues including skeletal muscle [7]. It enhances neovascularisation, and thus improves tissue oxygenation [22].

The macrophage migration inhibitory factor (MIF) is considered as an inflammatory mediator associated with metabolic diseases and can signal molecular links between adipocytes and myocytes. It has been proposed to have a role in myoblast differentiation but this role is still indistinct [28].

Brain-derived neurotrophic factor (BDNF) is now a well-known neurotrophine that regulates various neuronal processes [18]. It is recently suggested to play a vital role in metabolic regulation of skeletal muscle and in controlling energy homeostasis [29].

The improving effects of voluntary exercise on physical health have been proved [1], but contradictory results are raised about the ideal intensity, duration, and type of exercise required to get the favourable effects and avoid the bad effects of the high intensity exercise on systemic inflammation [11,22]. This study investigated whether the type of physical exercise, voluntary or forced, would induce different morphological changes in the structure of the skeletal muscles, in their myokines immuno-expression and in the serum corticosterone level, aiming to define the right way for the use of exercise as a therapeutic measure in different diseases.

\section{MATERIALS AND METHODS}

\section{Experimental animals}

Thirty-six adult male Wistar albino rats weighing 180-230 g were used in this study. The animals were kept under constant environmental and nutritional conditions throughout the study in metal cages (4 rats in each cage) in the behaviour lab (College of Medicine, Taibah University), exposed to a light/ /dark cycle at $14: 10$ hour photo cycle at $22-24^{\circ} \mathrm{C}$ with a free access to food and water and were observed for normal movement for 7 days. The animals were cared for in accordance with the National Institutes of Health guide for the care and use of Laboratory animals (NIH Publications No. 8023) and the protocol was approved by the Faculty Experimental Ethics Committee.

The rats were randomly divided into three groups (12 rats each):

- Control group: were kept on a standard chow diet for 3 weeks;

- Voluntary exercise group: were allowed to voluntary running (voluntary wheel running) for 3 weeks;

- Forced exercise group: were kept on a forced swimming regimen for 3 weeks.

\section{Voluntary exercise (running) regimen}

The rats were housed individually in cages equipped with rat running wheels (Lafayette Instrument Company, Inc. 3700 Sagamore Parkway North Lafayette, IN 47904 USA), and allowed to voluntary running for 3 weeks. The running wheels were tested every other day to confirm their proper function and the mean running distances were recorded for each rat [14].

\section{Forced exercise (swimming) regimen}

The rats were individually forced to swim inside vertical glass cylinders $(60 \mathrm{~cm}$ in diameter, $80 \mathrm{~cm}$ in depth) with no possibility of escape. The water depth was $50 \mathrm{~cm}$ maintained at a $25-28^{\circ} \mathrm{C}$. Swimming was conducted $15 \mathrm{~min}$ daily for 21 days. After each session of swimming, the rats were removed, allowed to dry and returned to their cages $[9,20]$. The distances-swum by each rat were recorded automatically via computer software (Ethovision XT version 8.0, Noldus Information Technology, Nieuwe Kanaal, 5 Wageningen, The Netherlands). 
Table 1. Mean weight of the rats before and after the experiment $(n=12)^{*}$

\begin{tabular}{lccccccc}
\hline Weight [g] & \multicolumn{3}{c}{ Group } & & \multicolumn{2}{c}{ ANOVA } \\
\cline { 2 - 3 } \cline { 6 - 7 } & Control & Voluntary exercise (running) & Forced exercise (swimming) & & F value & P value $^{* *}$ \\
\hline Weight before & $229.17 \pm 3.22$ & $235.42 \pm 0.82$ & $230.83 \pm 1.90$ & & 2.144 & 0.13 \\
Weight after & $273.67 \pm 7.18$ & $272.75 \pm 1.60$ & $272.08 \pm 4.38$ & & 0.26 & 0.97 \\
\hline
\end{tabular}

${ }^{*}$ Values are presented as means \pm standard error of mean, ${ }^{* *}$ No significant difference between different groups

Recording the weight and the corticosterone levels

The weight of each rat was recorded at the beginning of the experiment and before sacrifice.

On sacrifice (at $8 \mathrm{am})$, blood samples were collected from the thoracic cavity to quantify the serum corticosterone level. The sera was separated and stored at $-80^{\circ} \mathrm{C}$ till the corticosterone levels were assessed by using ELISA kits (ALPCO Diagnostics, Orangeburg, NY, USA) according to the manufacturers' instructions [4].

\section{Muscle sample collection} and histological preparation

Twenty-four hours after the last day of the experiment, the animals were sacrificed by cervical dislocation [20], the hind limbs were dissected and the main bulks of the gastrocnemius muscles were removed. Longitudinal and transverse sections were prepared from the muscles for paraffin sections, and stained with haematoxylin and eosin (H\&E) for light microscopic structural study via Bright field Automated microscope (Olympus BX 36).

Serial paraffin sections $(4 \mu \mathrm{m})$ were stained using primary polyclonal anti-rat antibodies against the chosen muscle myokines (Uscn Life Science Business Co., Ltd.; polyclonal Rat IL-6 PAA079Ra71, Rat VEGF PAA143Ra71, Rat MIF PAA698Ra01, Rat BDNF PAA011Ra01) and for Proliferating Cell Nuclear Antigen (PCNA; Ab-1 clone PC 10 mouse monoclonal antibody) to evaluate the proliferating muscle satellite cells [13]. The immunoperoxidase technique with a Bench-Mark instrument (Ventana Company) was used for immunostaining [12].

A light microscopic examination of the immuno-expression was performed and the immunohistochemical staining of myokines was evaluated by measuring the optical densities (ODs) of the stained sections. Briefly, 5 non-overlapping representative fields were captured at a magnification of $\times 200$ with a microscopic field area of $786.432 \mathrm{~m}^{2}$. The
ImageJ (NIH) software, version 2.0.0-beta4 (National Institutes of Health, Bethesda, MD, USA) was used for evaluation of the OD of immuno-stain regarding Verghese et al. [26] as follows: deconvolution of the image using diaminobenzidine (DAB) vector into three different coloured images, calibration of the brown DAB image by measuring the mean integrated intensities (mean grey value) of 5 non-overlapping zones of the stained tissue and then the intensity numbers were transformed into the OD using the following equation: $[O D=\log$ (max intensity/mean intensity)], where the max intensity $=250$, and the mean intensity $=$ mean grey value [26]. The PCNA stained cells (proliferating cells) were counted in 5 non overlapping fields $(\times 400)$ of the stained sections of the different groups for statistical analysis.

\section{Statistical analysis}

Statistical analysis was performed using SPSS V.23 (IBM Corporation, Somers, NY, USA). Values are presented as means \pm standard error of mean (SEM). Statistical evaluation was done using one-way analysis of variance (ANOVA) followed by Bonferroni pairwise comparisons and the level of significance was determined to be less than 0.05 throughout the study. Pearson's test was used for determining the correlation of distances and the $p$ and $r$ values were recorded.

\section{RESULTS}

The weight and corticosterone level

The mean weight of the rats before and after the experiment showed no significant difference between the groups (Table 1).

The corticosterone level showed no significant difference between the control and running groups ( $p=0.937$ ) whereas a highly significant increase in the levels of corticosterone were found between the control and swimming and between running and swimming groups ( $p<0.001$; Fig. 1 ). 


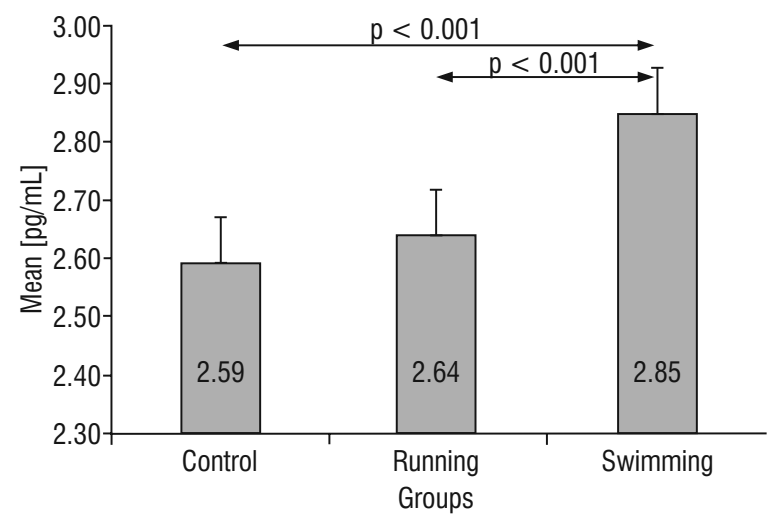

Figure 1. The mean serum corticosterone levels in different groups.

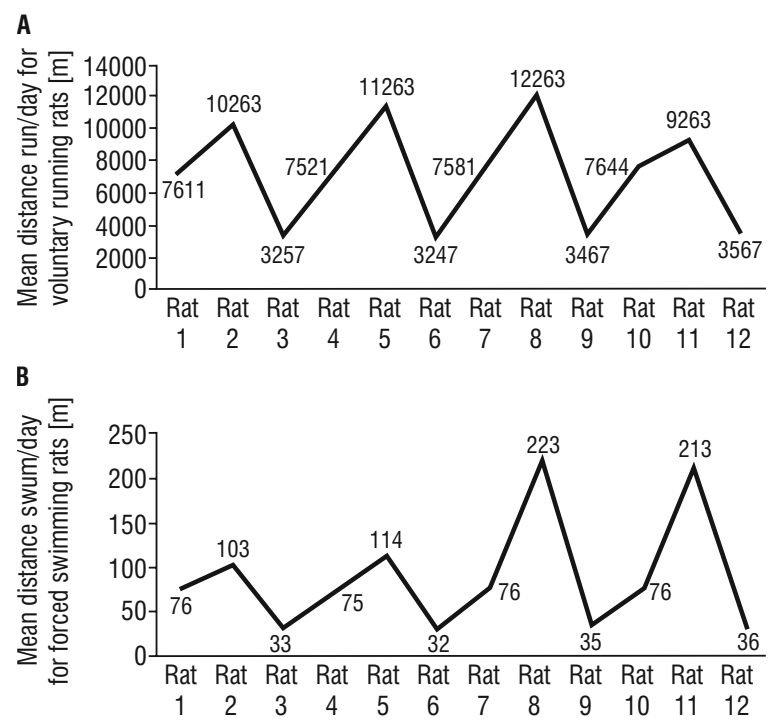

Figure 2. The mean distance run by the voluntary exercised rats (A), and the mean distance swum by the forced exercised rats (B).

\section{The mean distance run or swum by the rats}

The mean distance run by the voluntary running rats was $7253.92 \pm 935.38 \mathrm{~m} /$ day while the mean distance swum by the forced exercise group was $72.67 \pm 9.38 \mathrm{~m} /$ day (Fig. 2). Except for the detected significant correlation between the mean distance run and the OD value of VEGF $(r=0.867, p<0.001)$, no significant correlation was detected between the means of distance run or distance swum and any of the OD values of all analysed myokines.

\section{Histopathological and immunohistochemical analysis}

The control gastrocnemius muscle sections revealed a well-organised bundles of muscle fibres sepa- rated by scanty perimysial connective tissue contained thin walled muscular blood vessels. The myofibres showed a well-defined myofibril with peripheral dark stained nuclei, and few lightly stained satellite cells (Fig. 3A-C).

Sections of voluntary running rats showed bundles of hypertrophied muscle fibres with wide perimysial spaces containing fine collagen fibres. Nearly all the myofibres exhibited slight shearing with prominent transverse unstained banding of $Z$ Lines striation. The lightly stained satellite cells were dominating and can be identified from the dark stained myofibre nuclei. Slightly increased perivascular connective tissue and some venous congestion were observed (Fig. 3D-F).

Sections of forced exercise rats showed sporadic widely spaced bundles of muscle fibres with marked perimysial fibrosis. The individual fibres appeared atrophied, less in size and widely spaced compared to other groups, whether few fibres appeared hypertrophied. Most fibres lost the normal myofibril appearance and possessed homogenous acidophilic cytoplasm. The muscular blood vessels appeared with thick walls, marked perivascular fibrosis and inflammatory cell infiltrate. There were focal areas of necrosis with cellular infiltration and loss of the normal myofibre striations. The pale satellite cell nuclei are hardly seen (Fig. 3G-I).

The IL-6 immuno-stain expression showed marked increase and diffuse distribution in most muscle fibres of the voluntary running group. A marked decrease or absence of the stain was observed in the forced exercise muscle fibres as compared to control group (Fig. 4A-C).

The VEGF immuno-stain expression showed irregular distribution of the stain in the voluntary running group; it appeared to be increased in some fibres and markedly decreased in others. The stain was markedly decreased in nearly most fibres of the forced swimming group as compared to control group (Fig. 4D-F).

The MIF immuno-stain expression, though was difficult to identify, but it appeared to be increased in many fibres of the voluntary running group, but markedly decreased in the forced swimming group (Fig. 4G-I).

The BDNF immuno-stain expression was clearly identified with marked increase in nearly all muscle fibres and in satellite cells of voluntary running group, and marked decrease in the forced swimming group (Fig. 4J-L).

Voluntary exercise group showed significant increase of mean OD values of IL-6, MIF, and BDNF, 


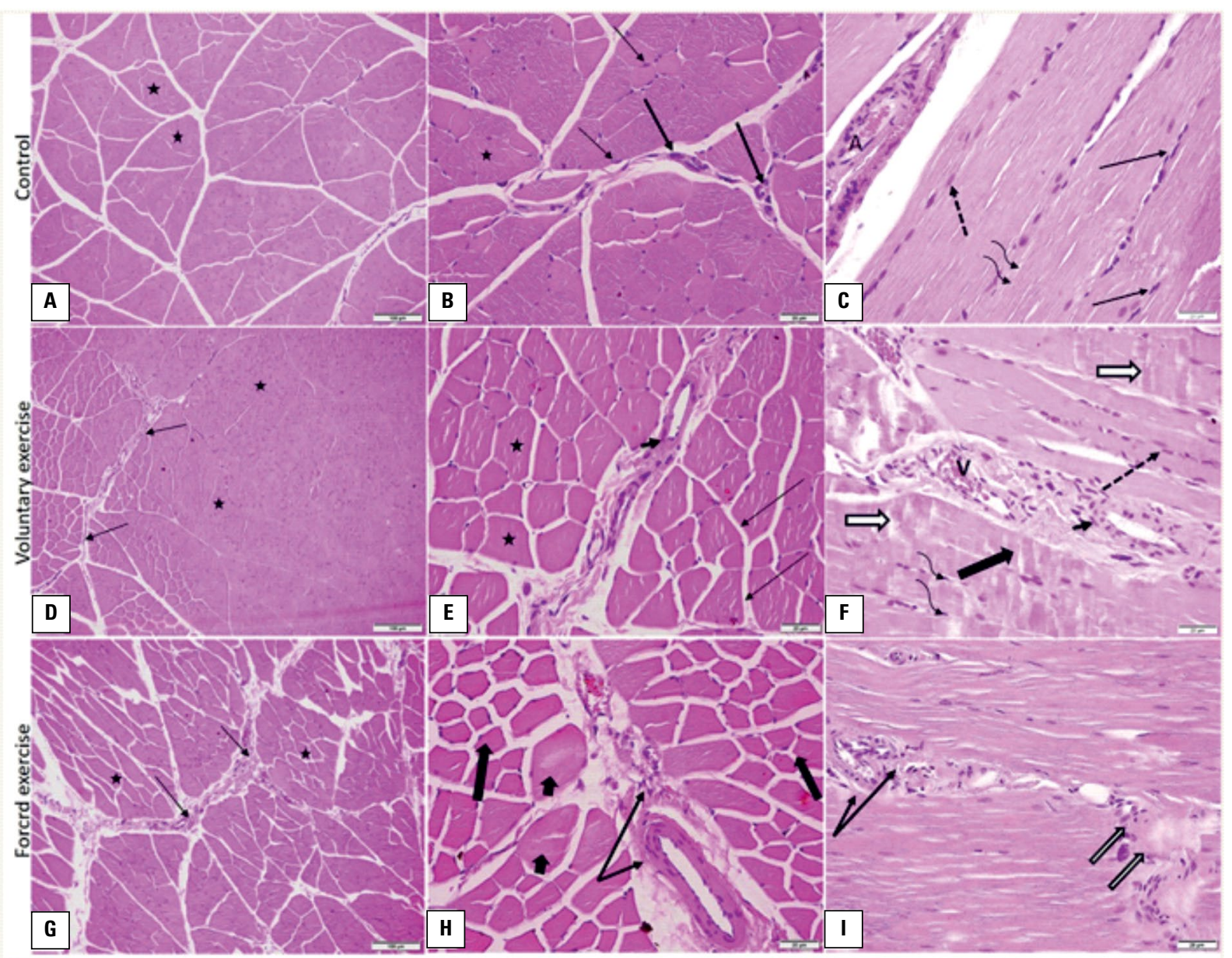

Figure 3. Digital photomicrographs of sections in the gastrocnemius muscle. 1. Control group (A, B, C) show well organised bundles of muscle fibres (asterisk) separated by scanty perimysial connective tissue. The myofibres show well defined myofibrils (curved arrows), peripheral dark stained nuclei (thin black arrows) and few lightly stained satellite cells (dotted arrows). A muscular arteriole (A) appears with normal wall thickness. The perimysium around the bundles contains thin walled blood vessels and scanty fibrous tissue (thick black arrows). 2. Voluntary running group (D, E, F) show bundles of hypertrophied muscle fibres (compared to those of control) (asterisk) separated by wide perimysial spaces containing fine collagen fibres (thin arrows). Nearly all the myofibres show slight shearing with noticeable myofibrils (thick arrow) and prominent transverse unstained banding of $Z$ Lines striation (white arrows). The lightly stained satellite cells are dominating (dotted arrows) and can be identified from darkly stained myofibre nuclei (curved arrow). Apparent slight increase in perivascular connective tissue (short thick arrows) and slight venous congestion (V) are observed. 3. Swimming group (G, H, I) show widely spaced bundles of muscle fibres (asterisk) with marked perimysium fibrosis (thin black arrows). Individual fibres looked spaced and small in size compared to other groups. Groups of muscle fibres appear atrophied with apparent small diameter (thick arrow), but few appeared hypertrophied (short thick arrows). Most fibres lost the normal myofibril appearance and possessed homogenous acidophilic cytoplasm. Muscular blood vessels appear with thick walls, marked perivascular fibrosis and inflammatory cell infiltrate (double arrows). Focal areas of necrosis, loss of normal myofibre striations and cellular infiltration are apparent (white arrows); haematoxylin and eosin stain; A, D, G: $\times 100 ; B, C, E, F, H, I: \times 400$.

whereas the forced exercise group showed significant decrease in their values compared to the control group. On the other hand, the OD of VEGF showed a non-significant decrease in the voluntary running group and a significant decrease in the forced exercise group as compared to the control one (Table 2).

The mean number of PCNA stained satellite cells were significantly higher in voluntary exercise group
(5.5 \pm 1.31$)$, and significantly lower in the forced exercise group $(0.83 \pm 0.72)$ as compared to that of the control group $(1.83 \pm 0.72)$ (Figs. 5,6 ).

\section{DISCUSSION}

The non-significantly changed mean weight of the animals in both types of exercise was consistent with results of Arnold and Salvatore [3]. 


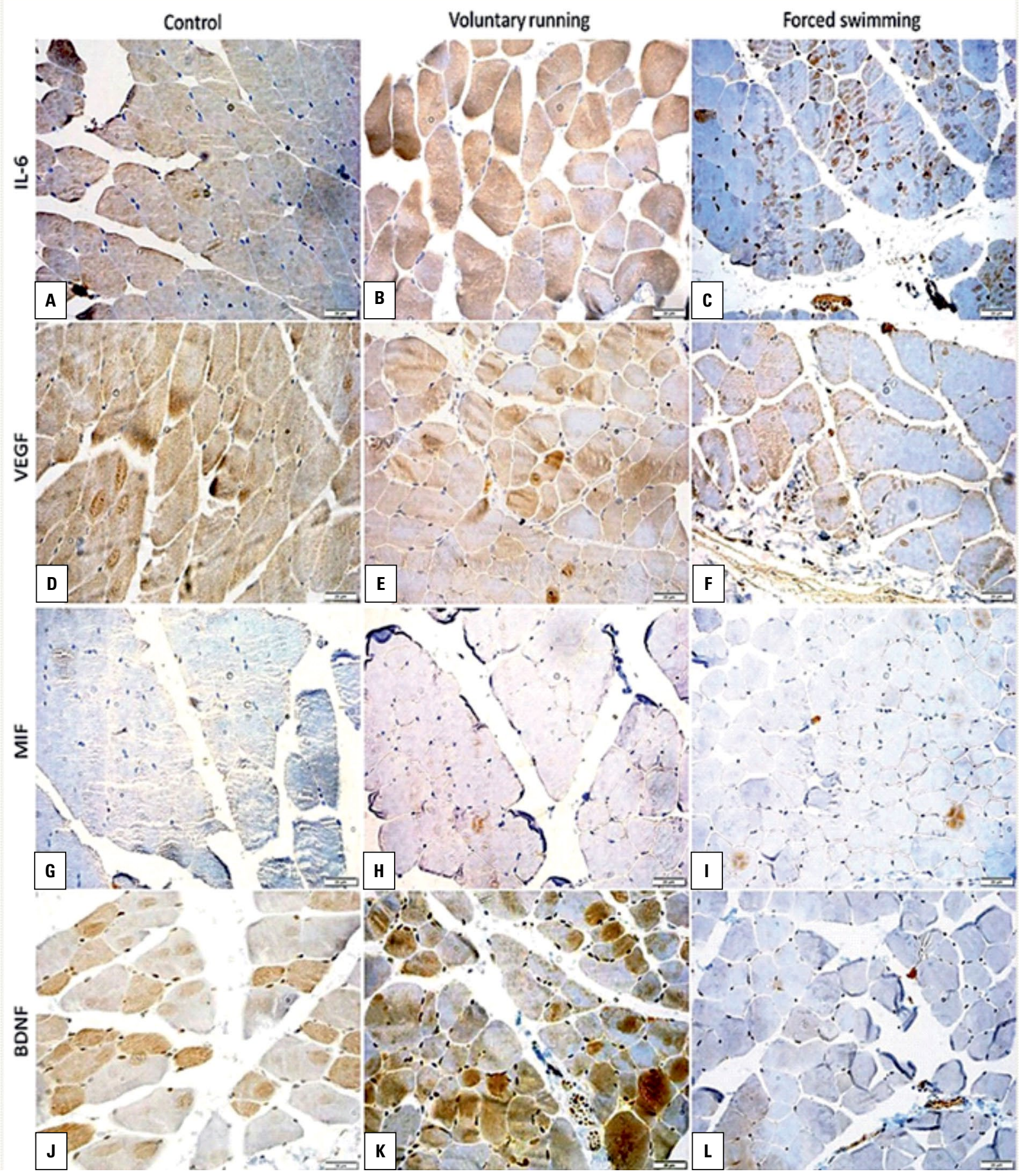

Figure 4. Digital photomicrographs of gastrocnemius muscle immunostained sections of different myokines in all groups show: 1. Marked increase and diffuse expression of interleukin 6 (IL-6) immunostaining in the voluntary running group muscle fibres, and marked decrease and irregularly distributed expression in the forced exercise fibres (as compared to control sections) (A, B, C); 2. Irregular expression of vascular endothelial growth factor (VEGF) stain in the voluntary running group appears to be increased in some fibres and markedly decreased in others as compared to control. The expression appears markedly decreased in nearly most fibres of the forced swimming group (D, E, F); 3. The migration inhibitory factor (MIF) exhibit some increase in many fibres of the voluntary running group and marked decrease in the forced swimming group (G, H, I); 4. Brain-derived neurotrophic factor (BDNF) immune expression show marked increase in most muscle fibres and in satellite cells of the voluntary running group and marked decrease or absent in the fibres of the forced swimming group $(\mathbf{J}, \mathbf{K}, \mathbf{L})$. 
Table 2. Mean optical density of the 4 myokines in all groups of rats $(n=12)^{*}$

\begin{tabular}{|c|c|c|c|c|c|}
\hline \multirow[t]{2}{*}{ Myokine } & \multicolumn{3}{|c|}{ Group } & \multicolumn{2}{|c|}{ ANOVA } \\
\hline & Control & Voluntary exercise (running) & Forced exercise (swimming) & F value & $P$ value \\
\hline \multirow[t]{3}{*}{ IL-6 } & $0.1095 \pm 0.006$ & $0.1643 \pm 0.013$ & $0.251 \pm 0.004$ & 70.652 & $\mathrm{P} 1<0.001$ \\
\hline & & & & & $\mathrm{P} 2<0.001$ \\
\hline & & & & & P3 $<0.001$ \\
\hline \multirow[t]{3}{*}{ VEGF } & $0.1849 \pm 0.007$ & $0.1672 \pm 0.006$ & $0.0778 \pm 0.006$ & 73.844 & $\mathrm{P} 1=0.212$ \\
\hline & & & & & $\mathrm{P} 2<0.001$ \\
\hline & & & & & $\mathrm{P} 3<0.001$ \\
\hline \multirow[t]{3}{*}{ MIF } & $0.0189 \pm 0.003$ & $0.0374 \pm 0.006$ & $0.0002 \pm 0.002$ & 22.469 & $\mathrm{P} 1=0.006$ \\
\hline & & & & & $P 2=0.006$ \\
\hline & & & & & $\mathrm{P} 3<0.001$ \\
\hline \multirow[t]{3}{*}{ BDNF } & $0.0699 \pm 0.007$ & $0.1870 \pm 0.025$ & $0.0060 \pm 0.026$ & 37.677 & $\mathrm{P} 1<0.001$ \\
\hline & & & & & $P 2=0.014$ \\
\hline & & & & & P3 $<0.001$ \\
\hline
\end{tabular}

*Values are presented as means \pm standard error of mean; BDNF — brain-derived neurotrophic factor; IL-6 — interleukin 6; MIF — migration inhibitory factor; VEGF — vascular endothelial growth factor; P1 — the significance between control and voluntary exercise groups; P2 — the significance between control and forced exercise groups; P3 — the significance between voluntary exercise and forced exercise groups

A

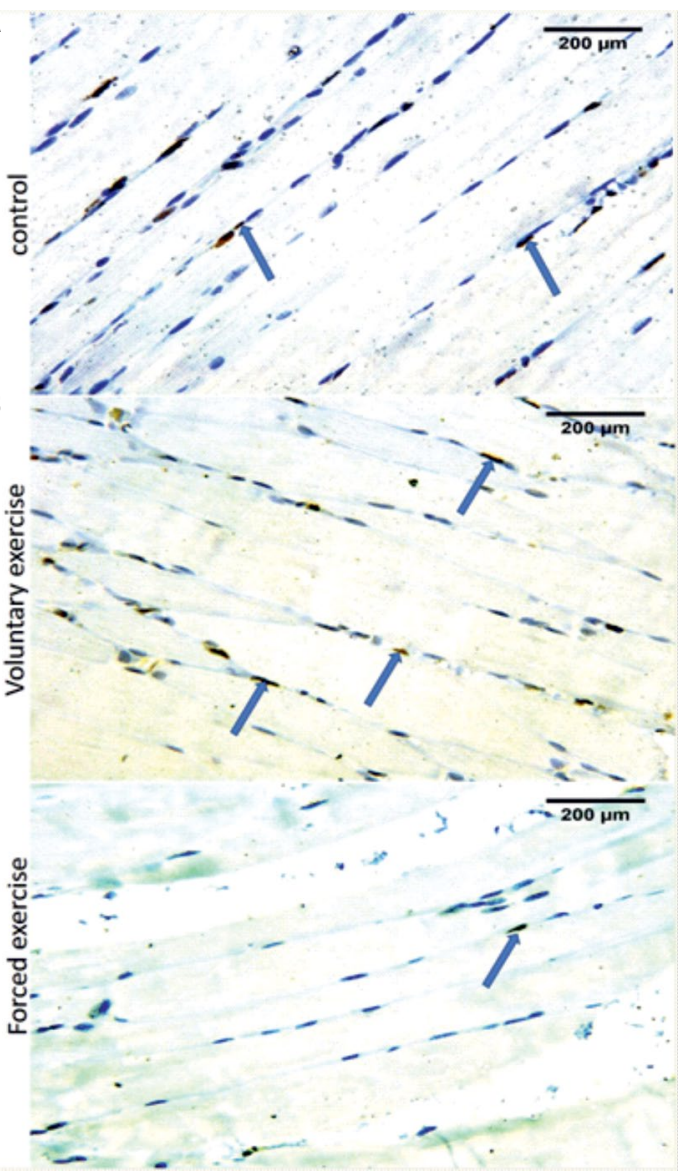

Figure 5. Proliferating cell nuclear antigen stained skeletal muscle satellite cells in control $(\mathbf{A})$, voluntary exercise $(\mathbf{B})$ and forced exercise (C) groups.

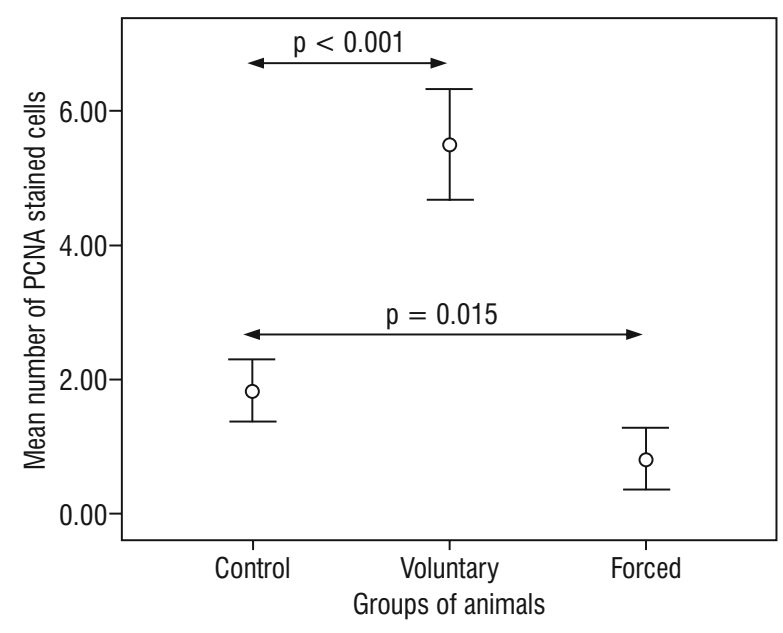

Figure 6. Error bar showing the number and significance of proliferating cell nuclear antigen (PCNA) stained cells in different groups.

The non-significant change of corticosterone levels in the voluntary running group could affirm the non-occurrence of stress after voluntary exercise regimen, whereas its significant increase after forced swimming regimen can support the occurrence of stress and/ depression after forced swim exercise $[8,9]$. This could be due to the gradual acclimatisation to exercise in voluntary running that minimise the acute psychological and physiological stress to which the rats are exposed in acute exposure to exercise [27]. 
Inconsistent reports exist on the responses of skeletal muscle to different modes of exercise [5]. In this study, we hypothesized that skeletal muscle would react to voluntary exercise as a physiological stimulus increasing the function by hypertrophy, but the forced exercise would be a harmful pathological stimulus. Voluntary wheel running caused hypertrophy of muscle fibres with prominent transverse banding and increased number of activated satellite cells which can denote that voluntary running can represent an appropriate regimen for satellite cells activation, proliferation, and/or differentiation with the resultant increased muscle mass [5].

After the forced swimming regimen, there were atrophy of muscle fibres with focal areas of necrosis, cellular infiltration and decreased number of activated satellite cells. In accordance with the present results, forced exercise adopted by treadmill, caused an immediate localised degenerative effect and late macrophage infiltration and necrosis of the deep slow antigravity muscle fibres [2]. Interestingly, they proved that changing the load laid on the muscle using the same type of exercise can evoke different degenerative muscle effects [2].

That practicing exercise can activate systemic intercellular and inter-organ communication via secreting the cytokines or myokines has raised the importance of studying these factors as therapeutic targets [21]. The significant increase and diffuse distribution of IL-6 immunostaining in the muscle fibres after voluntary running can denote its local production by different types of skeletal muscle fibres and can prove its positive role in the physiology of hypertrophic muscle growth [16]. It is important to consider that the local production of IL-6 in voluntary running muscle fibres might be transient and of short-term action, but it imposes important autocrine benefits either as an anti-inflammatory factor or as a regulator of glucose uptake and energy metabolism $[9,17]$. This action is different from the action of the long-lasting increased serum IL- 6 levels that are associated with inflammatory conditions and accompanied with increased muscle wasting and atrophy [16].

The significantly decreased and irregularly distributed immunostained IL- 6 in the forced exercise fibres can display the absence of a local beneficial or harmful effect of IL- 6 after the forced exercise regimen. This can be attributed to the rise of endogenous glucocorticoid production due to stress of the forced exercise which can also give explanation for the atrophy detected in groups of muscle fibres [15].

It has been increasingly clear that, in response to exercise, a skeletal muscle sometimes secretes profuse amounts of angiogenic factors, including VEGF that may function primarily as paracrine factors, but a controversial debate exists over the ideal amount of exercise needed for their beneficial effects [6]. The non-increase of VEGF immunostain after voluntary running observed in this study could be due to non-regular expression in all muscle fibres, as VEGF is expressed only in the predominantly glycolytic fibres. Also, there might be the hypoxia level needed for the generation and regulation of VEGF is not reached during the voluntary running regimen $[10,25]$. However, the lone significant correlation of the OD of VEGF expression with the mean distance run can prove the increased VEGF expression in the skeletal muscles in voluntary exercise but not in forced exercise.

The significant decrease of VEGF after the forced swimming exercise could be due to the inhibitory effect of stress of the forced regimen and the accompanying raised serum corticosterone level causing inhibition of endogenous VEGF production [15]. This coincided with the observed necrosis in some areas of the skeletal muscles due to the decreased basal skeletal muscle capillarisation with the resultant muscle cell apoptosis, and inhibition of angiogenesis during this type of exercise [24].

The study showed significant increase of the immunostain and OD values of both MIF and BDNF in the muscle fibres of the voluntary running rats. This is in accord with the recently proved elevated serum levels of both myokines by voluntary wheel running [9]. The concomitant relation of both myokines could lie in their suggested effects on the myogenic cells as both are expressed in the myoblasts and are modified during myoblast differentiation [28].

The decreased immuno-expression of both MIF and BDNF in the muscle fibres after forced exercise might be due to the psychological stress as denoted by the increased serum corticosterone level after the swimming regimen. Their decreased levels are consistent with the decreased serum levels of both myokines in forced swim-induced depression $[9,19]$.

\section{CONCLUSIONS}

The voluntary and forced exercises have different effects on the structure and myokines expression in 
skeletal muscle tissue and also on the serum corticosterone level. Forced exercise could be harmful to the muscle fibres and it decreases the myokines production, whereas voluntary running can increase the muscle mass and progenitor satellite cells. The use of exercise for the attainment of healthy life style or in psycho- or neuro-therapy should follow a thoroughly studied programme for welfare of human health.

\section{Acknowledgements}

The authors express thanks for the technicians of the behavioural lab, College of Medicine, Taibah University, who helped us throughout conduction of the experiment.

\section{REFERENCES}

1. Adlard PA, Perreau VM, Pop V, et al. Voluntary exercise decreases amyloid load in a transgenic model of Alzheimer's disease. J Neurosci. 2005; 25(17): 4217-4221, doi: 10.1523/JNEUROSCl.0496-05.2005, indexed in Pubmed: 15858047.

2. Armstrong RB, Ogilvie RW, Schwane JA. Eccentric exercise-induced injury to rat skeletal muscle. J Appl Physiol Respir Environ Exerc Physiol. 1983; 54(1): 80-93, doi: 10.1152/jappl.1983.54.1.80, indexed in Pubmed: 6826426.

3. Arnold JC, Salvatore MF. Getting to compliance in forced exercise in rodents: a critical standard to evaluate exercise impact in aging-related disorders and disease. J Vis Exp. 2014(90), doi: 10.3791/51827, indexed in Pubmed: 25178094.

4. Ayuob NN, Ali SS, Suliaman M, et al. The antidepressant effect of musk in an animal model of depression: a histopathological study. Cell Tissue Res. 2016; 366(2): 271-284, doi: 10.1007/s00441-0162468-9, indexed in Pubmed: 27481508.

5. Bazgir B, Fathi R, Rezazadeh Valojerdi M, et al. Satellite cells contribution to exercise mediated muscle hypertrophy and repair. Cell J. 2017; 18(4): 473-484, doi: 10.22074/cellj.2016.4714, indexed in Pubmed: 28042532.

6. Bellamy LM. Temporal Pattern of Type li Fibre- Specific Satellite Cell Expansion To Exercise Correlates With Human Muscle Hypertrophy: Potential Role for Myostatin. 2012.

7. Clow C, Jasmin BJ. Brain-derived neurotrophic factor regulates satellite cell differentiation and skeltal muscle regeneration. Mol Biol Cell. 2010; 21(13): 2182-2190, doi: 10.1091/mbc.e10-020154, indexed in Pubmed: 20427568.

8. Contarteze RV, Manchado FD, Gobatto CA, et al. Stress biomarkers in rats submitted to swimming and treadmill running exercises. Comp Biochem Physiol A Mol Integr Physiol. 2008; 151(3): 415-422, doi: 10.1016/j.cbpa.2007.03.005, indexed in Pubmed: 17428717.

9. Eldomiaty MA, Almasry SM, Desouky MK, et al. Voluntary running improves depressive behaviours and the structure of the hippocampus in rats: A possible impact of myokines. Brain Res. 2017; 1657: 29-42, doi: 10.1016/j.brainres.2016.12.001, indexed in Pubmed: 27919728.

10. Gavin TP, Westerkamp LM, Zwetsloot KA. Soleus, plantaris and gastrocnemius VEGF mRNA responses to hypoxia and exercise are preserved in aged compared with young female C57BL/6 mice. Acta Physiol (Oxf). 2006; 188(2): 113-121, doi: 10.1111/j.17481716.2006.01609.x, indexed in Pubmed: 16948798.

11. Gleeson M. Immune function in sport and exercise. J Appl Physiol (1985). 2007; 103(2): 693-699, doi: 10.1152/japplphysiol.00008.2007, indexed in Pubmed: 17303714.
12. Grizzle WE. Special symposium: fixation and tissue processing models. Biotech Histochem. 2009; 84(5): 185-193, doi: 10.3109/10520290903039052, indexed in Pubmed: 19886755.

13. Johnson SE, Allen RE. Proliferating cell nuclear antigen (PCNA) is expressed in activated rat skeletal muscle satellite cells. J Cell Physiol. 1993; 154(1): 39-43, doi: 10.1002/jcp.1041540106, indexed in Pubmed: 8093452.

14. Jonsson J. Effect of voluntary exercise on BDNF/TrkB gene expression and alcohol intake Josefine Jonsson. Master Thesis. 2012.

15. Klein GL. The effect of glucocorticoids on bone and muscle. Osteoporos Sarcopenia. 2015; 1(1): 39-45, doi: 10.1016/j. afos.2015.07.008, indexed in Pubmed: 26557727.

16. Muñoz-Cánoves $P$, Scheele $C$, Pedersen BK, et al. Interleukin- 6 myokine signaling in skeletal muscle: a double-edged sword? FEBS J. 2013; 280(17): 4131-4148, doi: 10.1111/febs.12338, indexed in Pubmed: 23663276.

17. Pedersen BK, Steensberg A, Fischer $C$, et al. Searching for the exercise factor: is IL-6 a candidate? J Muscle Res Cell Motil. 2003; 24(2-3): 113-119, doi: 10.1023/a:1026070911202, indexed in Pubmed: 14609022

18. Perdiguero E, Ruiz-Bonilla V, Gresh L, et al. Genetic analysis of p38 MAP kinases in myogenesis: fundamental role of p38alpha in abrogating myoblast proliferation. EMBO J. 2007; 26(5): 1245-1256, doi: 10.1038/sj.emboj.7601587, indexed in Pubmed: 17304211.

19. Porsolt RD. Animal models of depression: utility for transgenic research. Rev Neurosci. 2000; 11(1): 53-58, doi: 10.1515/revneuro.2000.11.1.53, indexed in Pubmed: 10716655.

20. Rodriguez I, Diaz A, Vaamonde D. Assessment of the effect of prolonged forced swimming on CD-1 mice sperm morphology with and without antioxidant supplementation. Andrologia. 2016; 48(3): 277-281, doi: 10.1111/and.12443, indexed in Pubmed: 26032180.

21. Rowe GC, Safdar A, Arany Z. Running forward: new frontiers in endurance exercise biology. Circulation. 2014; 129(7): 798-810, doi: 10.1161/CIRCULATIONAHA.113.001590, indexed in Pubmed: 24550551.

22. Schnyder S, Handschin C. Skeletal muscle as an endocrine organ: PGC-1 $\alpha$, myokines and exercise. Bone. 2015; 80: 115-125, doi: 10.1016/j.bone.2015.02.008, indexed in Pubmed: 26453501.

23. So B, Kim HJ, Kim J, et al. Exercise-induced myokines in health and metabolic diseases. Integr Med Res. 2014; 3(4): 172-179, doi: 10.1016/j.imr.2014.09.007, indexed in Pubmed: 28664094.

24. Tang K, Breen EC, Gerber HP, et al. Capillary regression in vascular endothelial growth factor-deficient skeletal muscle. Physiol Genomics. 2004; 18(1): 63-69, doi: 10.1152/physiolgenomics.00023.2004, indexed in Pubmed: 15084712.

25. Tang K, Breen EC, Wagner $\mathrm{H}$, et al. HIF and VEGF relationships in response to hypoxia and sciatic nerve stimulation in rat gastrocnemius. Respir Physiol Neurobiol. 2004; 144(1): 71-80, doi: 10.1016/j. resp.2004.04.009, indexed in Pubmed: 15522704.

26. Varghese $F$, Bukhari $A B$, Malhotra $R$, et al. IHC Profiler: an open source plugin for the quantitative evaluation and automated scoring of immunohistochemistry images of human tissue samples. PLoS One. 2014; 9(5): e96801, doi: 10.1371/journal. pone.0096801, indexed in Pubmed: 24802416.

27. Webster I, Du Toit EF, Huisamen B. Peer reviewed Peer reviewed short communication the effect of long term swim training on physiological stress levels in the rat. Med Tech. 2010; 24(2): 37-40.

28. Wen F, Zheng J, Yu J, et al. Macrophage migration inhibitory factor in the regulation of myoblast proliferation and differentiation. Biosci Biotechnol Biochem. 2016; 80(7): 1313-1320, doi: 10.1080/09168451.2016.1153951, indexed in Pubmed: 26927414.

29. Wisse BE, Schwartz MW. The skinny on neurotrophins. Nat Neurosci. 2003; 6(7): 655-656, doi: 10.1038/nn0703-655, indexed in Pubmed: 12830151. 